\title{
The triple-mode pulsating variable V823 Cassiopeiae ${ }^{\star}$
}

\author{
J. Jurcsik ${ }^{1}$, B. Szeidl ${ }^{1}$, M. Váradi ${ }^{1,2}$, A. Henden ${ }^{3}, Z^{2}$. Hurta $^{4}$, B. Lakatos ${ }^{4}$, K. Posztobányi ${ }^{4}$, \\ P. Klagyivik ${ }^{4}$, and Á. Sódor ${ }^{1,4}$
}

\author{
1 Konkoly Observatory of the Hungarian Academy of Sciences. PO Box 67, 1525 Budapest, Hungary \\ e-mail: jurcsik@konkoly.hu \\ 2 Department of Experimental Physics and Astronomical Observatory, University of Szeged, 6720 Szeged, Dóm tér 9, Hungary \\ 3 Universities Space Research Association/US Naval Observatory, PO Box 1149, Flagstaff, 86002-1149, Arizona, USA \\ e-mail: aah@nofs . navy.mil \\ 4 Eötvös Loránd University, Department of Astronomy, PO Box 32, 1518 Budapest, Hungary
}

Received 14 February 2005 / Accepted 23 August 2005

\begin{abstract}
Using extended multicolour CCD photometry of the triple-mode radial pulsator V823 Cas we studied the properties of the coupling frequencies invoked by nonlinear processes. Our results support that a resonance connection affects the mode coupling behaviour. The P1/P0 period ratio of V823 Cas has an "out of range" value if compared with the period ratios of the known double mode pulsators, while the P2/P1 period ratio is normal. The periods and period ratios cannot be consistently interpreted without conflict with pulsation and/or evolution models. We describe this failure with the suggestion that at present, the periods of V823 Cas are in a transient, resonance affected state, thus do not reflect the true parameters of the object. The anomalous period change behaviour of the fundamental and second overtone modes supports this idea. We have also raised the possibility that a $f_{0}+f_{2}=2 f_{1}$ resonance may act in triple mode pulsators.
\end{abstract}

Key words. stars: individual: V823 Cas - stars: variables: Cepheids - stars: oscillations - stars: evolution - techniques: photometric

\section{Introduction}

Stellar pulsation often shows multiperiodic behaviour. The simultaneous excitation of different modes is a common property of e.g. $\delta$ Sct variables. In these stars both radial and nonradial pulsation modes can be excited, and especially in case of a dense frequency spectrum with many nonradial modes, the identification of the modes still has not been settled.

Multiperiodicity of radial mode pulsators is also observed in different types of variables. Radial mode pulsating variables are important targets of astero-seizmology as mode identification is unambiguous in many cases. Consequently, the pulsation properties of these stars reveal the physical parameters quite precisely by using pulsation models and/or empirical relations developed for the different types of variables along the instability strip. Two radial modes can be excited simultaneously in Cepheids, HADS (high amplitude $\delta$ Sct stars) SX Phe and RR Lyrae variables. These so-called double mode pulsators oscillate either in the fundamental and first overtone modes or in the first and second overtones.

In three stars, AC And, V823 Cas and V829 Aql, the three lowest radial modes have been detected to be excited

* Tables 1-5 are only available in electronic form at the CDS via anonymous ftp to cdsarc.u-strasbg.fr $(130.79 .128 .5)$ or via http://cdsweb.u-strasbg.fr/cgi-bin/qcat?J/A+A/445/617 simultaneously. Very recently two triple-mode short period Cepheids were also discovered in the LMC (Moskalik \& Dziembowski 2005), these stars oscillate, however, in the first three overtone modes. As a result of the mass photometry of the LMC and SMC (OGLE, MACHO, EROS projects, Udalski et al. 1999; Soszyński et al. 2000; Alcock et al. 1999; Beaulieu et al. 1997) dozens of short period $(P<1 \mathrm{~d})$ double mode Cepheids were discovered, both fundamental and first overtone $(F U / F O)$ and first and second overtone $(F O / S O)$ pulsators. These results have also shown that in the LMC and SMC most of the $P<1 \mathrm{~d}$ Cepheids have double mode properties, indicating that in their physical parameter regimes multi-mode pulsation is favoured. Because of the large distance and the consequent faintness of the LMC/SMC multi-mode pulsators, their investigations are strongly restricted. The three galactic triplemode radial pulsators are also sparsely examined, even though our knowledge of stellar pulsation requires such studies.

According to their fundamental periods, AC And and V823 Cas $\left(P_{0} \sim 0.7 \mathrm{~d}\right)$ resemble metal-poor RR Lyrae stars, while V829 Aql with $P_{0}=0.29 \mathrm{~d}$ is classified as a post main sequence $\delta$ Sct star by Handler et al. (1998). The metal content of AC And is, however, extremely high for a typical RR Lyrae star (Preston 1959). Fitch \& Szeidl (1976) and Kovács \& Buchler (1994) based on pulsation and evolution models have concluded that it is most probably a higher mass evolved star 
similar to $\delta$ Sct variables. Fernie (1994) suggested that AC And may be the missing link between $\delta$ Sct stars and classical Cepheids.

V823 Cas was discovered to be a triple mode radial pulsator (Antipin 1997) using the Moscow photographic plate collection taken with the $40 \mathrm{~cm}$ astrograph in Crimea between 1948 and 1995. Data showed light variation with three independent periodicities matching most probably the three lowest radial mode oscillations. Until today, no further information on this important object has emerged, therefore, we decided to carry out an extensive multicolour photometric investigation of V823 Cas.

\section{Observations and data reduction}

The observations were obtained with the automated $60 \mathrm{~cm}$ telescope of the Konkoly Observatory (Svábhegy, Budapest) equipped with a Wright 750x1100 CCD (parameters and calibration are given in Bakos 1999) using $B V(R I)_{\mathrm{c}}$ filters. The field of view was $17^{\prime} \times 24^{\prime}$. Reduction processes were performed using standard IRAF $^{1}$ packages. Data were corrected for atmospheric extinction.

About 3600 frames were obtained in each passband on 38 nights between 25 September and 14 December in 2003 (JD 2452 908-2 452 988). Transformation to the standard system was done using the $B, V, R_{\mathrm{c}}, I_{\mathrm{c}}$ magnitudes of surrounding stars observed by A. Henden with the USNO Flagstaff Station $1.0 \mathrm{~m}$ telescope equipped with a SITe/Tektronix $1024 \times$ 1024 CCD. A complete table of the positions and $B V(R I)_{\mathrm{c}}$ magnitudes of stars in the field centred on V823 Cas, is given in Table 1 (available electronically from the CDS). The following formulae were derived to transform the instrumental data to the standard system:

$$
\begin{aligned}
& V=0.989 v+0.095(b-v) \\
& B=0.987 b-0.032(b-v) \\
& R_{\mathrm{c}}=0.986 r+0.112(v-r) \\
& I_{\mathrm{c}}=0.989 i-0.012(v-i) .
\end{aligned}
$$

Aperture photometry was applied to measure the relative magnitudes of V823 Cas to GSC 04018-01777 ( $V=11$ m $235, B-$ $\left.V=1.173, V-R_{\mathrm{c}}=0.617, V-I_{\mathrm{c}}=1.202\right)$. HD 134 , GSC 04018-01891 and GSC 04018-01661 were used as check stars, none of them showed any significant variability relative to GSC 04018-01777.

Photometric data are available electronically at the CDS. In Table 2-5, Col. 1 lists the HJD of the observations, and Column 2 gives the differential magnitudes of V823 Cas with respect to GSC 04018-01777 for the $B, V, R_{\mathrm{c}}, I_{\mathrm{c}}$ colours. $B-V$, $V-R_{\mathrm{c}}$, and $V-I_{\mathrm{c}}$ colour curves were also derived utilizing the $V$ observations and fitted values of the $B, R_{\mathrm{c}}, I_{\mathrm{c}}$ curves according to the Fourier solutions given in Tables 6 and 7 for the moments of the $V$ measurements. The colour indices are given in Table 3.

${ }^{1}$ IRAF is distributed by the National Optical Astronomy Observatories, which are operated by the Association of Universities for Research in Astronomy, Inc., under cooperative agreement with the National Science Foundation.

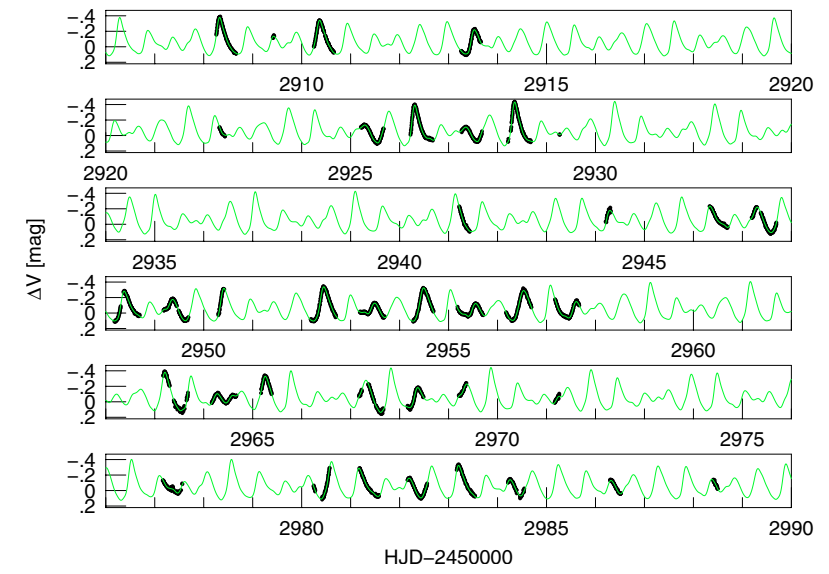

Fig. 1. $V$ light curve of V823 Cas fitted with the Fourier solution given in Tables 6 and 7.

\section{Photometric results}

\subsection{Frequency components, amplitudes and phases}

The light curve solution given in Tables 6 and 7 was determined from the Fourier analysis of the photometric data using the utilities of the program package MUFRAN (Kolláth 1990). Nonlinear regression facilities of Mathematica (Wolfram Research, Inc) were also applied. The listed frequencies fit the $B, V, R_{\mathrm{c}}, I_{\mathrm{c}}$ data with $0.0126,0.0079,0.0081$, 0.0079 mag rms scatter, respectively, which is about the observational accuracy limit. The $V$ light curve and the fit are shown in Fig. 1.

Besides the frequencies of the three dominant radial modes and their harmonics, 26 of the possible linear combination coupling frequencies could be identified in the successively prewhitened spectra of the $B$ data.

Frequency component identification was performed on the $B$ dataset as the amplitudes of the signals are the largest in $B$. In order to refine the $f_{0}, f_{1}, f_{2}$ frequency values least squares solutions were calculated within the vicinity of the suspected values of $f_{0}, f_{1}, f_{2}$, their harmonics and the detected linear combination term frequencies. Linear combination frequencies were always set according to the actual values of the main frequency components. The solution with the smallest residual scatter was accepted for the $f_{0}, f_{1}, f_{2}$ frequency values. The $2 \sigma$ asymptotic error estimates of the corresponding periods are 0.000045 , 0.000013 and 0.000060 days, respectively.

The formal errors $(1 \sigma)$ of the amplitudes are about $0.2-$ $0.4 \mathrm{mmag}$, the errors of the phases of signals with amplitude larger than $1 \mathrm{mmag}$ are reliable within $0.1-10^{\circ}$.

All but $3 f_{1}-2 f_{0}$ 2-term coupling frequencies involving $f_{1}, 2 f_{1}, 3 f_{1}$, and $f_{0}, 2 f_{0}$ appear in the spectra, and six 3-term linear combination frequencies are also detected. The amplitudes of the coupling components are always smaller than that of its compounding frequencies with one exception. The amplitude of the $f_{1}+3 f_{0}$ component is higher than the amplitude of the $3 f_{0}$ in each colour. The $3 f_{0}$ harmonic component is only marginally present in the data, we report this component only because a real frequency component was found at $6.43481 \mathrm{c} / \mathrm{d}$, which we could interpret only as $f_{1}+3 f_{0}$. However, considering 
Table 6. Amplitudes of the detected frequencies in $B, V, R_{\mathrm{c}}, I_{\mathrm{c}}, B-V, V-R_{\mathrm{c}}, V-I_{\mathrm{c}}$.

\begin{tabular}{|c|c|c|c|c|c|c|c|c|c|c|}
\hline \multicolumn{3}{|c|}{ Identification } & Frequency & $A_{B}$ & $A_{V}$ & $A_{R_{\mathrm{c}}}$ & $A_{I_{\mathrm{c}}}$ & $A_{B-V}$ & $A_{V-R_{\mathrm{c}}}$ & $A_{V-I_{\mathrm{c}}}$ \\
\hline & & & cycle/day & \multicolumn{7}{|c|}{ mmag } \\
\hline - & $f_{1}$ & - & 1.950515 & 176.45 & 130.23 & 102.37 & 79.59 & 46.42 & 28.01 & 51.22 \\
\hline - & $2 f_{1}$ & - & 3.901030 & 37.15 & 28.19 & 21.97 & 17.17 & 8.77 & 6.31 & 11.09 \\
\hline - & $3 f_{1}$ & - & 5.851545 & 13.13 & 10.25 & 7.67 & 6.96 & 3.00 & 2.54 & 3.25 \\
\hline - & $4 f_{1}$ & - & 7.802060 & 3.62 & 3.08 & 2.52 & 2.27 & 0.50 & 0.60 & 1.11 \\
\hline$f_{0}$ & - & - & 1.494765 & 115.95 & 86.00 & 67.10 & 52.28 & 30.17 & 19.26 & 34.60 \\
\hline $2 f_{0}$ & - & - & 2.989530 & 16.64 & 12.28 & 9.78 & 8.09 & 4.41 & 2.49 & 4.20 \\
\hline $3 f_{0}$ & - & - & 4.484295 & 2.14 & 1.30 & 0.88 & 0.99 & 1.31 & 1.29 & 1.31 \\
\hline- & - & $f_{2}$ & 2.433590 & 29.16 & 22.04 & 17.67 & 13.57 & 7.21 & 4.37 & 8.47 \\
\hline$f_{0}$ & $f_{1}$ & - & 3.445280 & 44.77 & 34.02 & 26.97 & 20.54 & 11.23 & 6.87 & 13.22 \\
\hline$-f_{0}$ & $f_{1}$ & - & 0.455750 & 25.10 & 19.45 & 15.45 & 12.15 & 5.67 & 4.02 & 7.28 \\
\hline $2 f_{0}$ & $f_{1}$ & - & 4.940045 & 11.76 & 8.79 & 7.30 & 5.99 & 3.17 & 1.47 & 2.74 \\
\hline $2 f_{0}$ & $-f_{1}$ & - & 1.039015 & 5.26 & 3.72 & 3.74 & 2.68 & 1.70 & 0.44 & 1.01 \\
\hline $3 f_{0}$ & $f_{1}$ & - & 6.434810 & 3.44 & 2.12 & 1.56 & 1.56 & 1.28 & 0.62 & 0.77 \\
\hline- & $f_{1}$ & $f_{2}$ & 4.384105 & 10.11 & 8.07 & 6.31 & 4.43 & 2.29 & 1.79 & 3.73 \\
\hline - & $-f_{1}$ & $f_{2}$ & 0.483075 & 6.94 & 5.43 & 3.88 & 3.35 & 1.43 & 1.64 & 2.13 \\
\hline$f_{0}$ & $f_{1}$ & $f_{2}$ & 5.878870 & 4.87 & 3.18 & 1.93 & 2.39 & 1.69 & 1.40 & 0.96 \\
\hline$-f_{0}$ & $f_{1}$ & $f_{2}$ & 2.889340 & 4.16 & 3.21 & 2.73 & 2.06 & 0.93 & 0.63 & 1.21 \\
\hline$f_{0}$ & $-f_{1}$ & $f_{2}$ & 1.977840 & 4.16 & 3.24 & 2.52 & 2.15 & 1.19 & 0.94 & 1.55 \\
\hline $2 f_{0}$ & $f_{1}$ & $f_{2}$ & 7.373635 & 1.56 & 1.52 & 1.33 & 0.76 & 0.25 & 0.27 & 0.80 \\
\hline$f_{0}$ & $2 f_{1}$ & - & 5.395795 & 10.70 & 7.91 & 5.97 & 4.51 & 2.78 & 2.10 & 3.41 \\
\hline$-f_{0}$ & $2 f_{1}$ & - & 2.406265 & 13.50 & 10.48 & 8.67 & 6.56 & 2.97 & 1.88 & 4.00 \\
\hline $2 f_{0}$ & $2 f_{1}$ & - & 6.890560 & 3.82 & 3.05 & 2.27 & 1.89 & 0.68 & 1.03 & 1.31 \\
\hline$-2 f_{0}$ & $2 f_{1}$ & - & 0.911500 & 2.07 & 1.92 & 1.25 & 0.89 & 0.17 & 0.70 & 1.24 \\
\hline - & $2 f_{1}$ & $f_{2}$ & 6.334620 & 5.89 & 4.18 & 3.22 & 2.61 & 1.71 & 0.97 & 1.63 \\
\hline$f_{0}$ & $2 f_{1}$ & $f_{2}$ & 7.829385 & 2.52 & 2.09 & 1.56 & 1.46 & 0.42 & 0.55 & 0.95 \\
\hline$-f_{0}$ & $2 f_{1}$ & $f_{2}$ & 4.839855 & 2.11 & 1.14 & 0.73 & 0.22 & 1.31 & 0.33 & 0.76 \\
\hline$f_{0}$ & $3 f_{1}$ & - & 7.346310 & 6.33 & 4.80 & 3.56 & 3.20 & 1.65 & 1.19 & 1.64 \\
\hline$-f_{0}$ & $3 f_{1}$ & - & 4.356780 & 3.50 & 3.15 & 2.63 & 1.55 & 0.39 & 0.65 & 1.76 \\
\hline $2 f_{0}$ & $3 f_{1}$ & - & 8.841075 & 1.72 & 1.58 & 0.63 & 0.53 & 0.52 & 0.95 & 1.15 \\
\hline- & $3 f_{1}$ & $f_{2}$ & 8.285135 & 3.05 & 2.19 & 1.27 & 1.62 & 1.00 & 0.91 & 0.59 \\
\hline- & $3 f_{1}$ & $-f_{2}$ & 3.417955 & 2.16 & 1.57 & 1.56 & 1.29 & 0.50 & 0.19 & 0.31 \\
\hline$f_{0}$ & $4 f_{1}$ & - & 9.296825 & 2.89 & 1.82 & 1.65 & 1.25 & 1.19 & 0.29 & 0.58 \\
\hline$f_{0}$ & - & $f_{2}$ & 3.928355 & 6.01 & 4.22 & 3.17 & 3.36 & 1.88 & 1.11 & 0.80 \\
\hline$-f_{0}$ & - & $f_{2}$ & 0.938825 & 2.45 & 2.22 & 1.12 & 1.16 & 0.71 & 1.12 & 1.47 \\
\hline$f_{3}$ & - & - & 0.817000 & 4.02 & 2.27 & 1.87 & 1.43 & 1.73 & 0.49 & 0.89 \\
\hline
\end{tabular}

the marginal amplitude of the $3 f_{0}$ component, this interpretation might not be correct.

After the removal of the radial modes, their harmonics and the coupling terms from the data, the residual spectra in each colour show evidence of a component at $0.817 \mathrm{c} / \mathrm{d}$ as shown in Fig. 2. This frequency, denoted as $f_{3}$ in Tables 6 and 7, cannot be resolved as the linear combination of the radial modes. As no indication of this frequency was found in the comparison - check stars data, we consider it as a real frequency component of V823 Cas. Taking into account the small amplitude and the relatively long $1.22 \mathrm{~d}$ periodicity of this variation one may speculate about its origin as a high-order gravity mode, as in the $\gamma$ Dor variables. However, further observational and theoretical confirmations are needed.

No other frequency in the residual spectrum was detected, consequently the solution given in Tables 6 and 7 is a full description of the data within the limits of the accuracy of the observations.

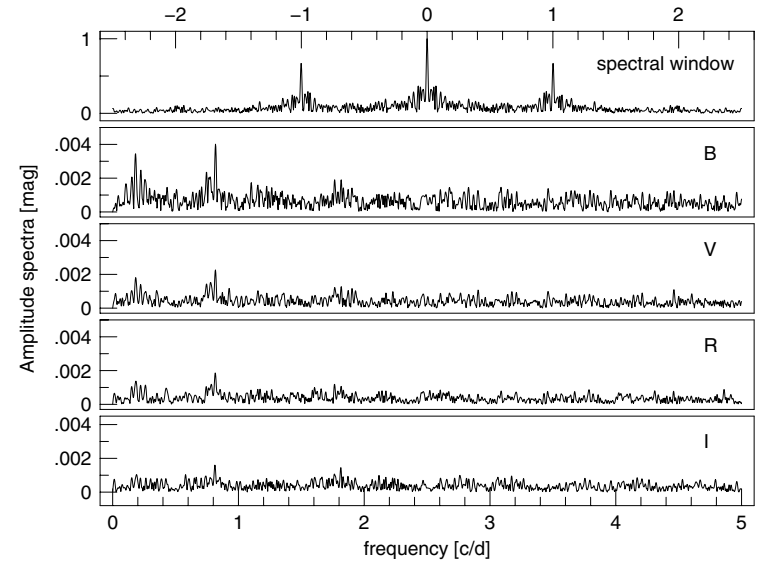

Fig. 2. Residual spectra of the $B, V, R_{\mathrm{c}}, I_{\mathrm{c}}$ data after prewhitening with the frequencies of the three radial modes, their harmonics and the linear combinations of these frequencies appearing in the Fourier spectra. In each colour the highest signal appears at $0.817 \mathrm{c} / \mathrm{d}$ indicating the detection of a real frequency component. 
Table 7. Phases of the detected frequencies in $B, V, R_{\mathrm{c}}, I_{\mathrm{c}}, B-V, V-R_{\mathrm{c}}, V-I_{\mathrm{c}}$ using cosine term Fourier sums and a $T_{0}=2452908$ initial epoch value.

\begin{tabular}{|c|c|c|c|c|c|c|c|c|c|c|}
\hline \multicolumn{3}{|c|}{ Identification } & \multirow{2}{*}{$\frac{\text { Frequency }}{\text { cycle/day }}$} & $\Phi_{B}$ & $\Phi_{V}$ & $\Phi_{R_{\mathrm{c}}}$ & $\Phi_{I_{\mathrm{c}}}$ & $\Phi_{B-V}$ & $\Phi_{V-R_{\mathrm{c}}}$ & $\Phi_{V-I_{\mathrm{c}}}$ \\
\hline & & & & \multicolumn{7}{|c|}{ deg } \\
\hline- & $f_{1}$ & - & 1.950515 & -85.24 & -86.21 & -87.87 & -90.72 & -82.60 & -80.09 & -79.14 \\
\hline - & $2 f_{1}$ & - & 3.901030 & 17.17 & 16.42 & 15.56 & 14.11 & 20.43 & 18.79 & 19.82 \\
\hline - & $3 f_{1}$ & - & 5.851545 & 167.96 & 165.83 & 165.24 & 167.21 & 179.39 & 166.14 & 161.51 \\
\hline- & $4 f_{1}$ & - & 7.802060 & -40.83 & -40.81 & -36.70 & -24.62 & -46.76 & -57.82 & -74.12 \\
\hline$f_{0}$ & - & - & 1.494765 & -9.53 & -11.77 & -14.50 & -18.37 & -3.11 & -2.26 & -1.81 \\
\hline $2 f_{0}$ & - & - & 2.989530 & 220.48 & 218.75 & 217.74 & 215.09 & 225.52 & 222.46 & 225.57 \\
\hline $3 f_{0}$ & - & - & 4.484295 & 69.47 & 66.47 & 57.45 & 58.57 & 247.29 & 66.61 & 66.60 \\
\hline- & - & $f_{2}$ & 2.433590 & -32.64 & -33.35 & -34.37 & -34.62 & -30.66 & -29.49 & -31.23 \\
\hline$f_{0}$ & $f_{1}$ & - & 3.445280 & 156.53 & 156.86 & 157.49 & 157.50 & 156.46 & 154.16 & 155.79 \\
\hline$-f_{0}$ & $f_{1}$ & - & 0.455750 & 124.69 & 124.51 & 123.20 & 125.22 & 124.11 & 130.23 & 123.71 \\
\hline $2 f_{0}$ & $f_{1}$ & - & 4.940045 & 16.59 & 18.51 & 20.08 & 21.15 & 7.98 & 14.15 & 14.22 \\
\hline $2 f_{0}$ & $-f_{1}$ & - & 1.039015 & 124.89 & 131.37 & 138.78 & 130.44 & 108.37 & 43.21 & 135.37 \\
\hline $3 f_{0}$ & $f_{1}$ & - & 6.434810 & 259.64 & 248.62 & 244.81 & 234.59 & 275.08 & 259.32 & 277.82 \\
\hline- & $f_{1}$ & $f_{2}$ & 4.384105 & 78.22 & 81.44 & 82.74 & 80.75 & 55.23 & 82.75 & 85.81 \\
\hline- & $-f_{1}$ & $f_{2}$ & 0.483075 & 234.69 & 235.40 & 230.99 & 238.92 & 229.87 & 246.51 & 230.05 \\
\hline$f_{0}$ & $f_{1}$ & $f_{2}$ & 5.878870 & -78.41 & -78.36 & -62.65 & -65.54 & -79.30 & -98.87 & -108.18 \\
\hline$-f_{0}$ & $f_{1}$ & $f_{2}$ & 2.889340 & 255.21 & 262.08 & 253.65 & 252.93 & 235.83 & 295.82 & 273.64 \\
\hline$f_{0}$ & $-f_{1}$ & $f_{2}$ & 1.977840 & 54.17 & 67.07 & 57.34 & 46.21 & 10.08 & 96.75 & 98.38 \\
\hline $2 f_{0}$ & $f_{1}$ & $f_{2}$ & 7.373635 & 119.60 & 131.29 & 137.83 & 145.01 & 44.71 & 91.55 & 116.02 \\
\hline$f_{0}$ & $2 f_{1}$ & - & 5.395795 & -80.14 & -77.50 & -70.74 & -72.81 & -88.61 & -96.49 & -83.44 \\
\hline$-f_{0}$ & $2 f_{1}$ & - & 2.406265 & 242.97 & 240.53 & 238.93 & 237.65 & 249.56 & 249.04 & 245.89 \\
\hline $2 f_{0}$ & $2 f_{1}$ & - & 6.890560 & 158.83 & 158.21 & 171.31 & 170.19 & 169.90 & 126.06 & 138.32 \\
\hline$-2 f_{0}$ & $2 f_{1}$ & - & 0.911500 & -3.69 & 2.88 & 3.42 & -27.58 & -77.19 & 0.01 & 22.05 \\
\hline- & $2 f_{1}$ & $f_{2}$ & 6.334620 & 224.63 & 224.04 & 228.02 & 217.36 & 225.35 & 209.85 & 234.81 \\
\hline$f_{0}$ & $2 f_{1}$ & $f_{2}$ & 7.829385 & 95.76 & 91.42 & 94.31 & 114.91 & 112.82 & 84.46 & 55.10 \\
\hline$-f_{0}$ & $2 f_{1}$ & $f_{2}$ & 4.839855 & 72.13 & 78.17 & 67.14 & 108.60 & 71.59 & 104.80 & 66.94 \\
\hline$f_{0}$ & $3 f_{1}$ & - & 7.346310 & 43.06 & 41.77 & 41.87 & 49.15 & 43.61 & 42.78 & 28.25 \\
\hline$-f_{0}$ & $3 f_{1}$ & - & 4.356780 & 30.46 & 39.13 & 33.64 & 38.47 & -44.35 & 54.05 & 35.88 \\
\hline $2 f_{0}$ & $3 f_{1}$ & - & 8.841075 & -81.99 & -67.51 & -65.18 & -37.29 & -147.19 & -68.90 & -79.12 \\
\hline- & $3 f_{1}$ & $f_{2}$ & 8.285135 & 29.87 & 20.70 & 18.68 & 21.75 & 54.73 & 21.53 & 13.96 \\
\hline- & $3 f_{1}$ & $-f_{2}$ & 3.417955 & 34.45 & 48.91 & 52.36 & 43.12 & -1.11 & -12.86 & 58.26 \\
\hline$f_{0}$ & $4 f_{1}$ & - & 9.296825 & 199.65 & 185.55 & 178.67 & 191.70 & 218.01 & 236.97 & 175.97 \\
\hline$f_{0}$ & - & $f_{2}$ & 3.928355 & 134.81 & 135.73 & 141.33 & 137.49 & 140.06 & 114.45 & 123.12 \\
\hline$-f_{0}$ & - & $f_{2}$ & 0.938825 & 171.24 & 155.66 & 169.61 & 197.53 & 222.10 & 141.50 & 124.41 \\
\hline$f_{3}$ & - & - & 0.817000 & 76.96 & 82.20 & 75.96 & 76.14 & 68.92 & 108.98 & 92.31 \\
\hline
\end{tabular}

In most cases the linear combination components involving negative terms have smaller amplitudes than the combination of the same positive terms, in agreement with model result, that nonlinear pulsation favours combination frequency terms where both $i$ and $j$ are positive (Antonello \& Aikawa 1998). There is one significant counter-example, $2 f_{1}-f_{0}$ has a larger amplitude than $2 f_{1}+f_{0}$ in each colour. The amplitudes of the 3-term combination frequencies of the same components have similar amplitudes independently of the signs.

The amplitude ratios of the combination frequencies defined as $R_{i j k}=(|i|+|j|+|k|) A_{i j k} /\left(|i| A_{100}+|j| A_{010}+|k| A_{001}\right)$ decrease exponentially with increasing order $(|i|+|j|+|k|)$ as shown in Fig. 3. $A_{i j k}$ denotes the amplitude of the $i f_{0}+j f_{1}+k f_{2}$ frequency component, i.e., $A_{100}=A\left(f_{0}\right)$, $A_{010}=A\left(f_{1}\right)$ and $A_{001}=A\left(f_{2}\right)$.

The amplitudes of all the detected frequencies decrease towards longer wavelengths. Most of the frequencies of the light

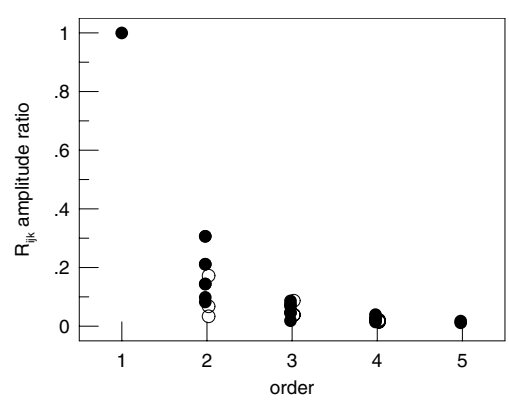

Fig. 3. Amplitude ratios of the different order combination frequency terms. Filled and open circles denote frequencies with positive $i, j, k$ values and involving negative terms as well, respectively. The decrease in the amplitude ratios is exponential with increasing order.

curve solution appear in the colour indices as well, typically with the largest amplitude in the $V-I_{\text {c }}$ colour. 
The phases of the three pulsation modes tend to be smaller towards the longer wavelengths. The phase differences between the $B$ and $I_{\mathrm{c}}$ data for $f_{0}, f_{1}$ and $f_{2}$ are $9^{\circ}, 5^{\circ}$ and $2^{\circ}$, respectively, while the phases of the colour indices are typically larger than the phases of the light curves, e.g. the phases of $f_{0}, f_{1}$ and $f_{2}$ of the $B-V$ curve are larger by about $8.7^{\circ}, 3.6^{\circ}$ and $2.7^{\circ}$ than the phases of the $V$ light curve solution. In contrast to these systematic trends of the phases of the three radial modes, the phases of the two largest amplitude linear combination terms, $f_{1}+f_{0}$ and $f_{1}-f_{0}$, are identical in each wavelength band. The phases of the smaller amplitude linear combination terms are not accurate enough to detect any real wavelength-dependent trend in the phase behaviour.

Poretti \& Pardo (1997) have shown that in galactic doublemode Cepheids the different order generalized phase differences $\left(G_{i j k}\right.$, for a definition see Antonello 1994) of the linear combination term frequencies tend to have similar values. In Table 8 the different order generalized phase differences of our $B$ light curve solution and also the $R_{i j k}$ amplitude ratios are listed, in the order of decreasing amplitude. The $1 \sigma$ errors of the phases are also given to easily estimate the reliability of the $G_{i j k}$ phase difference values. For compatibility with other similar data, the phase differences are given in radians. The averages of the $G_{i j k}$ phase differences are $3.55,1.07,5.01$ (5.26 omitting the most deviant 2.577 value), and 3.34 for the 2nd, 3rd, 4th and 5th order terms, respectively. These values are systematically smaller by about $0.5-1.0 \mathrm{rad}$ as the corresponding 4.31, 2.17, 6.24, 3.85 averages of the $G_{i j}$ values of galactic double-mode Cepheids calculated from the data listed by Poretti \& Pardo (1997). Taking into account the decreasing trend of the phase differences with decreasing periods as shown in Fig. 2 in Poretti \& Pardo (1997) the short periods of V823 Cas may account for its smaller $G_{i j k}$ values.

As indicated by Antonello \& Aikawa (1998) the only plausible explanation that can change the phases and can lead to coherent values of the different order phase differences is a resonance between the pulsation modes. In Fig. 4 the $B$ light curve is folded according to the periods of the three radial modes (top panels), after prewhitening with the frequencies and harmonics of the other two modes (middle panels), and the residual light curve after the removal of the three radial modes' light curves (bottom panels). These plots still show a regular behaviour according to the main periodicities. It is also evident that large amplitudes occur when each of the modes are simultaneously around maximum phase indicating that a resonance interaction may be responsible for the coupling frequencies.

\section{Comparison with model results}

Period ratios of different radial multi-mode pulsators have been collected (Fig. 5). The $\sim 0.01$ separations of the $P_{1} / P_{0}$ sequences of galactic, LMC and SMC Cepheids reflect the different metal content of the stars $(Z=0.02,0.008$ and 0.004 , respectively). The $P_{1} / P_{0}$ ratio of V823 Cas is evidently discrepant from the global $\log P_{1}-P_{1} / P_{0}$ relation defined by the different types of variables. This ismost likely because of an anomalous value of $P_{0}$, as the $P_{2} / P_{1}$ period ratio fits well the observed values of other multi-mode pulsators.
Table 8. Generalized phase differences and amplitude ratios of the frequencies of the $B$ light curve solution:

$G_{i j k}=\phi_{i j k}-\left(i \phi_{100}+j \phi_{010}+k \phi_{001}\right)$, $R_{i j k}=(|i|+|j|+|k|) A_{i j k} /\left(|i| A_{100}+|j| A_{010}+|k| A_{001}\right)$.

\begin{tabular}{rrrrrcccc}
\hline \hline Order & $i$ & $j$ & $k$ & Frequency & $A(B)$ & $R_{i j k}$ & Error $\left(\Phi_{i j k}\right)$ & $G_{i j k}$ \\
\hline 1 & 0 & 1 & 0 & 1.950515 & 0.1765 & 1.000 & 0.002 & 0.000 \\
& 1 & 0 & 0 & 1.494765 & 0.1160 & 1.000 & 0.003 & 0.000 \\
& 0 & 0 & 1 & 2.433590 & 0.0292 & 1.000 & 0.011 & 0.000 \\
\hline 2 & 1 & 1 & 0 & 3.445280 & 0.0448 & 0.307 & 0.008 & 4.384 \\
& 0 & 2 & 0 & 3.901030 & 0.0372 & 0.211 & 0.008 & 3.273 \\
& -1 & 1 & 0 & 0.455750 & 0.0251 & 0.172 & 0.013 & 3.496 \\
& 2 & 0 & 0 & 2.989530 & 0.0166 & 0.143 & 0.020 & 4.179 \\
& 0 & 1 & 1 & 4.384105 & 0.0101 & 0.099 & 0.035 & 3.421 \\
& 0 & -1 & 1 & 0.483075 & 0.0069 & 0.068 & 0.059 & 3.176 \\
& 1 & 0 & 1 & 3.928355 & 0.0060 & 0.083 & 0.055 & 3.087 \\
& -1 & 0 & 1 & 0.938825 & 0.0025 & 0.172 & 0.149 & 3.390 \\
\hline 3 & -1 & 2 & 0 & 2.406265 & 0.0135 & 0.087 & 0.025 & 0.763 \\
& 0 & 3 & 0 & 5.851545 & 0.0131 & 0.075 & 0.026 & 1.108 \\
& 2 & 1 & 0 & 4.940045 & 0.0118 & 0.086 & 0.028 & 2.109 \\
& 1 & 2 & 0 & 5.395795 & 0.0107 & 0.069 & 0.031 & 1.742 \\
& 0 & 2 & 1 & 6.334620 & 0.0059 & 0.046 & 0.059 & 1.179 \\
& 2 & -1 & 0 & 1.039015 & 0.0053 & 0.039 & 0.063 & 1.024 \\
& 1 & 1 & 1 & 5.878870 & 0.0049 & 0.046 & 0.069 & 0.855 \\
& -1 & 1 & 1 & 2.889340 & 0.0042 & 0.039 & 0.090 & 0.059 \\
& 1 & -1 & 1 & 1.977840 & 0.0042 & 0.039 & 0.080 & 0.194 \\
& 3 & 0 & 0 & 4.484295 & 0.0021 & 0.018 & 0.180 & 1.711 \\
\hline 4 & 1 & 3 & 0 & 7.346310 & 0.0063 & 0.039 & 0.054 & 5.378 \\
& 2 & 2 & 0 & 6.890560 & 0.0038 & 0.026 & 0.087 & 6.077 \\
& 0 & 4 & 0 & 7.802060 & 0.0036 & 0.021 & 0.093 & 5.236 \\
& -1 & 3 & 0 & 4.356780 & 0.0035 & 0.022 & 0.095 & 4.826 \\
& 3 & 1 & 0 & 6.434810 & 0.0034 & 0.026 & 0.094 & 6.510 \\
& 0 & 3 & 1 & 8.285135 & 0.0031 & 0.022 & 0.103 & 5.551 \\
& 1 & 2 & 1 & 7.829385 & 0.0025 & 0.020 & 0.124 & 5.380 \\
& 0 & 3 & -1 & 3.417955 & 0.0022 & 0.016 & 0.160 & 4.492 \\
& -1 & 2 & 1 & 4.839855 & 0.0021 & 0.017 & 0.162 & 4.635 \\
& -2 & 2 & 0 & 0.911500 & 0.0021 & 0.014 & 0.150 & 2.577 \\
& 2 & 1 & 1 & 7.373635 & 0.0016 & 0.014 & 0.211 & 4.475 \\
\hline 5 & 1 & 4 & 0 & 9.296825 & 0.0029 & 0.018 & 0.111 & 3.314 \\
& 2 & 3 & 0 & 8.841075 & 0.0017 & 0.011 & 0.193 & 3.363 \\
\hline & & & & & & & & \\
\hline
\end{tabular}

To investigate the anomalous value of the fundamental mode period first we have checked whether the observed periods and period ratios can match stellar pulsation and evolution model results. The difference between pulsation periods derived from linear models and the real nonlinear periods are supposed to be negligible. Thus linear periods are widely used to determine physical parameters of multimode pulsators (e.g. Moskalik \& Dziembowski 2005; Petersen \& Christensen-Dalsgaard 1996; Antonello \& Aikawa 1998). Kovács \& Buchler (1994) (hereafter KB94) calculated an extended set of linear radial models to explain the periods of AC And, the other triple-mode variable with very similar periods. We have compared the pulsation periods calculated from the KB94 formulae with those given in the evolutionary and pulsation models of (Alibert et al. 1999). The period of a given model agrees within $1 \%$ accuracy with the period calculated 


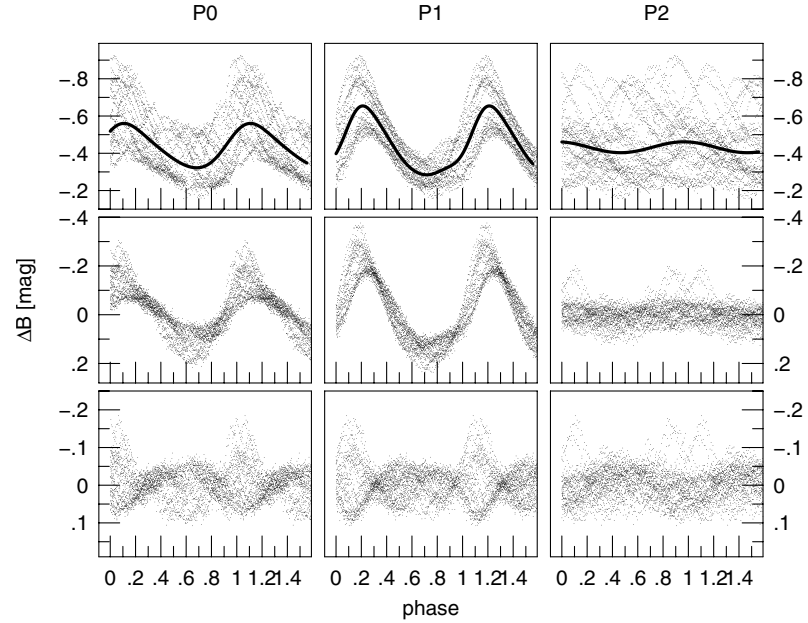

Fig. 4. $B$ light curve of V823 Cas folded with the periods of the three radial modes (top panels). The synthetic light curves corresponding to the three periods are also shown calculated from the amplitudes and phases of the three radial modes and their harmonics according to the data given in Tables 6 and 7. Middle panels show the folded light curves after subtracting the other two radial modes and their harmonics. The shape of the light curve of a given mode is strongly variable depending on the relative phases of the modes. In the bottom panels residual data after removing the light curves of the three radial modes are shown. These panels clearly indicate that the amplitude of the light variation increases significantly when each of the modes are simultaneously around maximum brightness. This enhancement may account for the numerous coupling frequencies appearing in the Fourier spectrum of the data in accordance with the model results of Antonello \& Aikawa (1998).

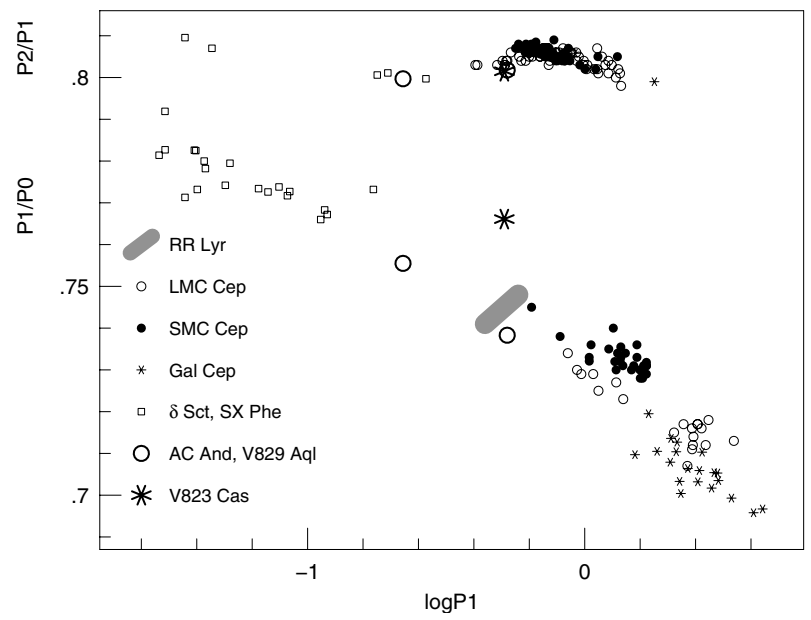

Fig. 5. Period ratios as a function of $\log P_{1}$ of different types of multi-mode radial pulsators. Though $P_{1}$ is the same for AC And and V823 Cas, their $P_{1} / P_{0}$ period ratios differ by about 0.03 which cannot be explained by any major difference in their physical parameters consistent with pulsation and evolutionary models.

from KB94 models (within the common parameter regimes), indicating the consistency of the two model groups.

The observed periods, and period ratios $P_{0}=0.669001 \mathrm{~d}$, $P_{1} / P_{0}=0.7663, P_{2} / P_{1}=0.8015$, can be fitted with period ratio distance $\Delta<0.003$ (for definition see KB94) accuracy with certain high-mass-sequence 2 models of KB94. Solutions

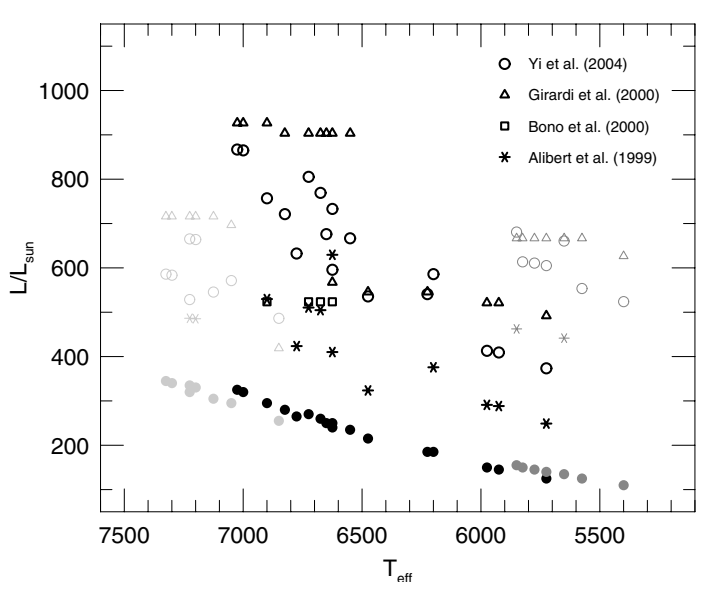

Fig. 6. Comparison of pulsation solutions for the observed periods and period ratios of V823 Cas with evolutionary model results. Filled circles denote pulsation solutions according to the KB94 models for $Z=0.004$ (light gray), $Z=0.01$ (black) and $Z=0.02$ (dark gray) compositions, respectively. Other symbols indicates the luminosities of evolutionary tracks during first crossing of the same mass and metallicity models at the temperature of the pulsation solutions. Second, third crossing luminosities are at even higher luminosities. The corresponding evolutionary models have the same mass within $\pm 0.25 M_{\odot}$ and the same metallicity within $Z= \pm 0.002$ ranges as the pulsation model solutions have. Each of the evolutionary models indicates higher luminosity than the pulsation solution.

can be found for the $Z=0.004$ and $Z=0.01$ models with 3.7-4.0 $M_{\odot}$ and 3.85-4.25 $M_{\odot}$ mass, respectively. The metalpoor solutions $(Z=0.004)$ are at too high temperatures $(T>$ $7000 \mathrm{~K}$ ), out of the instability strip, with negative growth rates of the detected modes, therefore we exclude these possibilities. There is also an indication that solutions might also exist for solar composition $Z=0.02$ models, but at "out of range" $M \sim 4.5$ mass values, which was not covered by the KB94 models, thus these pulsation solutions are somewhat uncertain. Moreover, considering the Period - Temperature connections valid for Cepheids, e.g., Fig. 1 in Baraffe \& Alibert (2001) and Fig. 3 in Sandage (2004), the temperature of Cepheids with periods shorter than 1 day must be higher than $6000 \mathrm{~K}$. All the $Z=0.02$ model period solutions fall below $6000 \mathrm{~K}$, thus these are not valid solutions either.

The parameter regimes of the possible solutions are shown in Fig. 6. The physical parameters of these models seem to be in conflict with evolutionary results. At a given mass and chemical composition any canonical evolutionary model predicts the lowest possible luminosity within the instability strip during its "first crossing", evolving off the main sequence. However, each of the appropriate evolutionary models (Alibert et al. 1999; Bono et al. 2000; Girardi et al. 2000; Yi et al. 2004) cross the instability strip at significantly higher luminosities than the solutions of the periods indicate even during the first crossing. The minimal luminosity at a given metallicity and mass values according to evolutionary models are also indicated in Fig. 6 . The 100-600 $L_{\odot}$ discrepancy between luminosities allowed by stellar evolution and luminosities derived from the pulsation solution of the periods also indicates that the periods of V823 Cas are anomalous. 
This problem arises from the unusually large value observed for the $P_{1} / P_{0}$ ratio (0.766). At $P_{0} \approx 0.7 \mathrm{~d}$ the normal value of $P_{1} / P_{0}$ would be less than 0.75 for any consistent model. The extended linear nonadiabatic pulsation models for galactic, LMC and SMC compositions (Morgan \& Welch 1997) strengthen that $P_{1} / P_{0}$ can never be larger than 0.75 in any real case. There are two ways to increase the period ratio at a given period, with metal poor models, and/or with models of smaller $L / M$ ratio the $P_{1} / P_{0}$ ratio is larger (Petersen $\&$ Christensen-Dalsgaard 1996; Kovács \& Buchler 1994). For V823 Cas, however, both of these possibilities can be excluded, as already mentioned the metal poor period solution falls out of the instability strip, and a small $L / M$ ratio is not allowed by evolutionary models.

Thus, we have found that the period ratios of V823 Cas cannot be explained consistently with stellar evolution and pulsation models. This is not the consequence of an inadequacy of the models but more probably arises from a temporal, transition behaviour of V823 Cas, which produces unusual period ratios (see the next section).

\section{Period changes}

The extended photographic data complemented with the recent CCD observations enable us to derive reliable period changes for the three observed modes. Antipin (1997) analysed two sets of the photographic data (between 2432 853-2 439145 and 2440 071-2 449633, respectively) and detected a period increase of the fundamental and first overtone modes. As photographic data are available from JD 2429 076, we divided the photographic data into three subsets to obtain more detail of the period change behaviour of V823 Cas. Table 9 lists the periods of the radial modes, their $2 \sigma$ error estimates and the photographic or $\operatorname{CCD}(B)$ amplitudes derived from the nonlinear regression process of Mathematica.

For comparison, the period change behaviour of AC And has been revised and listed in Table 9. Periods for the different datasets of AC And have been determined similarly as described in Sect. 3.1.

The period change rate of the dominant, first overtone mode of V823 Cas and that of the three modes of AC And correspond to the theoretically expected evolutionary value during the first crossing of the instability strip. The evolutionary period change rates during the second and third crossing are by two orders of magnitude slower (Moskalik \& Dziembowski 2005). The larger $\dot{P}_{1} / P_{1}$ indicates somewhat larger mass of V823 Cas than AC And.

In contrast, the periods of the fundamental mode and the second overtone of V823 Cas behave differently. According to the CCD observations the period change rates of these modes have been recently changed: $P_{0}$ has increased, while $P_{2}$ has decreased by about $0.0001 \mathrm{~d}$ during the last decades. The amplitude of the second overtone also shows significant changes: it has gradually decreased from $0.07 \mathrm{mag}$ to $0.03 \mathrm{mag}$, in contrast to the amplitudes of the fundamental and first overtone modes, which differ only within the limits of the uncertainty for the different sets of the observations. The reduction of the

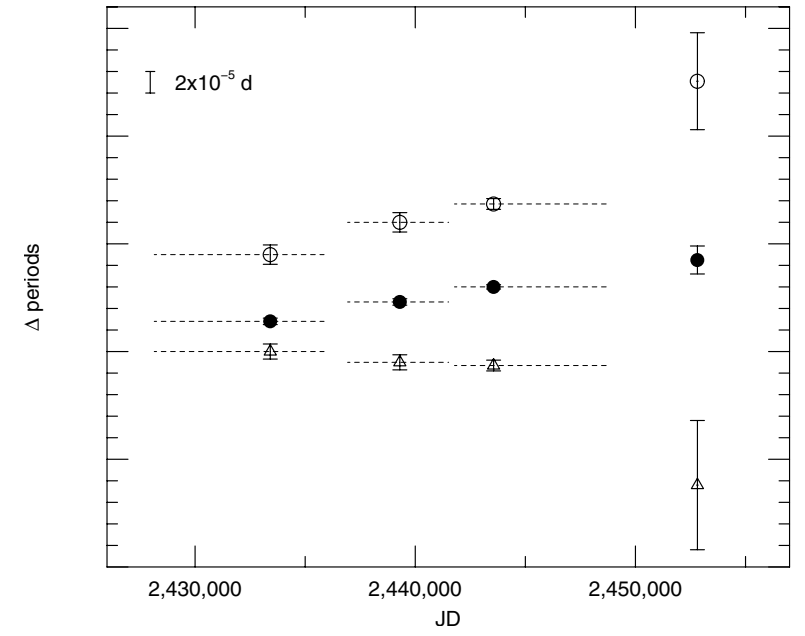

Fig. 7. Detected periods of V823 Cas at different epochs. $P_{0}-0.66854 \mathrm{~d}(\circ), P_{1}-0.51240 \mathrm{~d}(\bullet)$, and $P_{2}-0.41088 \mathrm{~d}(\triangle)$ are plotted for clarity.

$P_{2}$ amplitude indicates that this mode may be diminishing, and this explains its extreme period change behaviour.

An interesting possibility arises from the opposite sign period change of the second overtone mode. Extrapolating backward the linear period changes detected between JD $2430000-$ 2445000 , the period ratios should have been about $P_{1} / P_{0}=$ 0.76679 and $P_{2} / P_{1}=0.81089$ about 3300 years ago. These period ratios fulfill the $f_{0}+f_{2}=2 f_{1}$ resonance criterion, as $f_{0}=0.76679 f_{1}$ and $f_{2}=1.23321 f_{1}$, indicating that the three radial modes might have been in a resonance connection in the very recent astronomical past.

This resonance hypothesis would also help to understand the anomalous period ratios of V823 Cas. If we assume that in the future the period change rate of the first overtone remains the same as detected for the last 50 years, but the period increase of the fundamental remains greater than that, as indicated by recent observations, i.e.,

$\dot{P}_{1} / P_{1}-\dot{P}_{0} / P_{0}=C<0, C \sim$ const.

then this would lead to change in the period ratio, as

$\frac{\mathrm{d}\left(P_{1} / P_{0}\right)}{\mathrm{d} t}=P_{1} / P_{0} \times\left(\dot{P}_{1} / P_{1}-\dot{P}_{0} / P_{0}\right)=C \times P_{1} / P_{0}$.

Consequently, with time

$\left(P_{1} / P_{0}\right)_{t}=\left(P_{1} / P_{0}\right)_{t=0} \times \mathrm{e}^{C t}$.

The exponential change in the period ratio results in drastic changes during astronomically very short times. The recent observations have shown that the period change rate of $P_{0}$ has increased to 3 times that of the period change rate of $P_{1}$. Assuming the possible largest uncertainties, $C$ is smaller than -2 Myear $^{-1}$ at present, which, according to the above formula would lead to a decrease in the $P_{1} / P_{0}$ ratio from its unusual 0.766 to a normal 0.745 value within 14000 years. 
Table 9. Periods and period change rates of V823 Cas and AC And.

\begin{tabular}{|c|c|c|c|c|c|c|c|c|c|c|c|}
\hline Star & JD-2 400000 & $P_{0}[\mathrm{~d}]$ & $2 \sigma\left(P_{0}\right)$ & $\operatorname{mp}\left(P_{0}\right)$ & $P_{1}[\mathrm{~d}]$ & $2 \sigma\left(P_{1}\right)$ & $\operatorname{mp}\left(P_{1}\right)$ & $P_{2}[\mathrm{~d}]$ & \multicolumn{3}{|c|}{$2 \sigma\left(P_{2}\right) \operatorname{Amp}\left(P_{2}\right)$} \\
\hline \multicolumn{12}{|c|}{ V823 Cas } \\
\hline & $52908-52988$ & 0.669001 & .000045 & 0.12 & 0.512685 & .000013 & 0.18 & 0.410916 & .000060 & 0.03 & $\mathrm{CCD}(\mathrm{B})(1)$ \\
\hline & $42273-49033$ & 0.668887 & .000005 & 0.13 & 0.512660 & .000002 & 0.18 & 0.411027 & .000005 & 0.05 & $\operatorname{pg}(2)$ \\
\hline & $37575-42016$ & 0.668870 & .000009 & 0.12 & 0.512646 & .000003 & 0.17 & 0.411030 & .000007 & 0.05 & $\operatorname{pg}(2)$ \\
\hline & $29076-36540$ & 0.668840 & .000009 & 0.13 & 0.512628 & .000003 & 0.17 & 0.411040 & .000007 & 0.07 & pg (2) \\
\hline \multicolumn{12}{|c|}{ AC And } \\
\hline & $47864-49039$ & 0.711253 & .000014 & & 0.525155 & .000009 & & 0.421088 & .000014 & & Hip (3) \\
\hline & $36459-37949$ & 0.711232 & .000002 & & 0.525133 & .000001 & & 0.421072 & .000002 & & $\operatorname{pe}(\mathrm{V})(4)$ \\
\hline & $30258-35009$ & 0.711227 & .000001 & & 0.525130 & .000001 & & 0.421067 & .000001 & & $\operatorname{pg}(5)$ \\
\hline & $25540-27278$ & 0.711217 & .000011 & & 0.525108 & .000008 & & 0.421063 & .000016 & & vis (6) \\
\hline \multicolumn{2}{|c|}{ Period change rates } & $\dot{P}_{0} / P_{0}$ & & & $\dot{P}_{1} / P_{1}$ & \multicolumn{6}{|c|}{$\dot{P}_{2} / P_{2} \quad\left[\right.$ Myear $\left.^{-1}\right]$} \\
\hline \multicolumn{2}{|c|}{ V823 Cas } & increasing & & & 2.17 & \multicolumn{6}{|c|}{ decreasing } \\
\hline \multicolumn{2}{|c|}{ AC And } & 0.71 & & & 0.79 & \multicolumn{6}{|c|}{1.08} \\
\hline
\end{tabular}

References of the data: (1) this paper; (2) Antipin (1997); (3) ESA (1997); (4) Fitch \& Szeidl (1976); (5) Guman (1982); (6) Lause (1932, 1933, 1938).

\section{Absolute parameters derived from observed magnitudes, and $P-L$ relations}

The magnitude and intensity mean $V$ brightness and colours of V823 Cas using the standard magnitudes of the comparison star as given in Sect. 2 are the followings. Magnitude mean values:

$V=11.175, B-V=0.792, V-R_{\mathrm{c}}=0.473, V-I_{\mathrm{c}}=0.987$.

Intensity mean values:

$V=11.169, B-V=0.787, V-R_{\mathrm{c}}=0.471, V-I_{\mathrm{c}}=0.982$.

The interstellar reddening in the direction of V823 Cas according to the Schlegel et al. (1998) maps is quite large, $E(B-V)=0.887 \mathrm{mag}$. V823 Cas lies close to the galactic plane $\left(b=0.99^{\circ}\right)$ in the direction of the Perseus Arm. The distance of the Perseus Arm is about $3 \mathrm{Kpc}$ in the direction of V823 Cas $\left(l=118^{\circ}\right)$ as shown in Fig. 2 in Quillen (2002). Consequently, it can be assumed that within $3 \mathrm{Kpc}$ the interstellar reddening is much smaller than $0.887 \mathrm{mag}$. Dereddened colours, using the standard reddening law for $B V(R I)_{\mathrm{c}}$ colours as given in Schlegel et al. (1998), fit the synthetic colours of atmosphere models (Castelli et al. 1997) according to the parameters listed in Table 10.

The observed periods of V823 Cas are too short for classical Cepheids and are too long for $\delta$ Scuti models. This intermediate status makes comparison with model results uncertain, as in most of the cases conclusions can be drawn from extrapolation. Because of this deficiency and the uncertainties of data conversion between the observable (magnitude, colour-indices) and the theoretical plane's (luminosity, temperature) quantities, the absolute parameters from the observed colours and periods cannot be determined accurately.

A rough estimate can be, however, done by combining the possible solutions listed in Table 10 with predictions of empirical and theoretical Period - Luminosity relations. The
Table 10. Solutions for dereddening the observed colours of V823 Cas.

\begin{tabular}{ccccccl}
\hline \hline$E(B-V)$ & $Z$ & \multirow{2}{*}{$T_{\text {eff }}$} & \multicolumn{4}{c}{$M_{V}$} \\
& & & 1.5 & 2.0 & 2.5 & $3.0[\mathrm{Kpc}]$ \\
\hline 0.37 & 0.020 & 6500 & -0.93 & -1.56 & -2.04 & -2.44 \\
0.19 & 0.010 & 5750 & -0.35 & -0.98 & -1.46 & -1.86 \\
0.15 & 0.004 & 5500 & -0.22 & -0.85 & -1.33 & -1.73 \\
\hline
\end{tabular}

$0.669 \mathrm{~d}$ fundamental mode, and the $0.513 \mathrm{~d}$ first overtone periods correspond to $M_{V}=-0.9$ mag absolute visual magnitude during first crossing within \pm 0.3 mag range for $Z=0.02-0.004$ matallicity models according to the Period - Luminosity relations of Baraffe \& Alibert (2001) and Alibert et al. (1999). This value is in accordance with combined empirical $P-L$ relations of Cepheids and $\delta$ Scuti stars (e.g. Fernie 1992). Recent empirical studies e.g., Udalski (2000); Sandage (2004) also yield consistent results within the given magnitude range.

Comparing this value with the absolute magnitudes of different reddening and metallicity solutions at a distance of 1.5-3.0 Kpc as given in Table 10 we conclude that if the Period - Luminosity relation of classical Cepheids is valid for V823 Cas, it is either a $1.5 \mathrm{Kpc}$ distant, $T_{\text {eff }}=6500 \mathrm{~K} \mathrm{ob}-$ ject with solar composition or a cooler, $Z=0.01-0.004$ metallicity object at about $2 \mathrm{Kpc}$. As it was already mentioned in Sect. 4, Cepheids with $P<1$ day are hotter than $6000 \mathrm{~K}$, thus we conclude that the most probable solution for V823 Cas is $E(B-V)=0.37, d=1.5 \mathrm{Kpc}, T_{\text {eff }}=6500 \mathrm{~K}, M_{V}=-0.9$, $Z=0.02$. Because of the uncertainties involved, this result has to be taken with caution.

\section{Summary}

The recent CCD observations of V823 Cas can be fitted with the periods of the 3 lowest radial modes, their harmonics and 
their linear combination terms within the observational accuracy. No indication of nonradial p-modes has been found, but the probable existence of a small amplitude signal with $1.22 \mathrm{~d}$ periodicity needs an explanation.

We present, for the first time, extended multicolour observations of a multi-mode radial pulsator that are accurate enough to study the phase and amplitude relations of the radial modes and the different order coupling frequency components. These results give a deeper insight into the behaviour of the nonlinear processes taking place during stellar pulsation.

The observed period ratios of V823 Cas cannot be consistently explained with canonical pulsation and evolution models. We interpret this anomaly as a consequence of the temporal behaviour of the periods which may originate from very rapid evolutionary changes of the stellar parameters and/or a resonance connection between the radial mode frequencies $\left(f_{0}+f_{2}=2 f_{1}\right)$, in the astronomically recent past. Both theoretical and observational investigations are needed to verify this resonance during the triple-mode phase of the pulsation, and to examine its effect on the observed period values.

If the actual values of the $P_{1}$ and $P_{0}$ period change rates remain unchanged, a normal $P_{1} / P_{0}$ period ratio will be reached in about 10-20000 years. This peculiar period change behaviour, together with the lapse of the $P_{2}$ mode, as indicated by its amplitude decrease, may also hint that in its triple-mode state the periods of V823 Cas may have transient, resonanceaffected values, which do not reflect the real physical parameters of the star.

The unique period change behaviour of V823 Cas warns us about drawing conclusions about the evolutionary status and the direction of evolution from the period change rates. A similar conclusion was reached from the unexpected period change behaviour of the two recently-discovered overtone mode triple-mode radial pulsators in the LMC by Moskalik \& Dziembowski (2005).

Acknowledgements. We would like to thank Dr S. Antipin for kindly providing the photographic measurements of V823 Cas. This research has made use of the SIMBAD database, operated at CDS Strasbourg, France. The financial support of OTKA grants T-043504, T-046207 and T-048961 is acknowledged.

\section{References}

Alcock, C., Allsman, R. A., Alves, D. R., et al. 1999, ApJ, 511, 185

Alibert, Y., Baraffe, I., Hauschildt, P., \& Allard, F. 1999, A\&A, 344, 551

Antipin, S. 1997, A\&A, 326, L1

Antonello, E., \& Aikawa, T. 1998, A\&A, 337, 145

Antonello, E. 1994, A\&A, 291, 820

Bakos, G. Á. 1999, Occ. Techn. Notes Konkoly Obs., No. 11, http://www. konkoly.hu/mitteilungen/mitteilungen.html Baraffe, I., \& Alibert, Y. 2001, A\&A, 371, 592

Beaulieu, J.-P., Krockenberger, M., Sasselov, D., et al. 1997, A\&A, 321, L5

Bono, G., Caputo, F., Cassisi, S., et al. 2000, ApJ, 543, 955

Castelli, F., Gratton, R. G., \& Kurucz, R. L. 1997, A\&A, 318, 841

ESA 1997, The Hipparcos and Tycho Catalogues, ESA SP-1200

Fernie, J. D. 1992, AJ, 103, 1647

Fernie, J. D. 1994, MNRAS, 271, L19

Fitch, W. S., \& Szeidl, B. 1976, ApJ, 203, 616

Girardi, L., Bressan, A., Bertelli, G., \& Chiosi, C. 2000, A\&AS, 141, 371

Guman, I. 1982, Commun. Konkoly Obs., No. 78

Handler, G., Pikall, H., \& Diethelm, R. 1998, IBVS, No. 4549

Kolláth, Z. 1990, Occ. Techn. Notes Konkoly Obs., No. 1, http://www . konkoly.hu/staff/kollath/mufran.html

Kovács, G., \& Buchler, J. R. 1994, A\&A, 281, 749

Lause, F. 1932, Astron. Nachr., 245, 329

Lause, F. 1933, Astron. Nachr., 249, 377

Lause, F. 1938, Astron. Nachr., 264, 143

Morgan, S. M., \& Welch, D. L. 1997, AJ, 114, 1183

Moskalik, P., \& Dziembowski, W. 2005, A\&A, 434, 1077

Petersen, J. O., \& Christensen-Dalsgaard, J. 1996, A\&A, 312, 463

Poretti, E., \& Pardo, I. 1997, A\&A, 324, 133

Preston, G. W. 1959, ApJ, 130, 507

Quillen, A. C. 2002, AJ, 124, 924

Sandage, A., Tammann, G. A., \& Reindl, B. 2004, A\&A, 424, 43

Schlegel, D. J., Finkbeiner, D. P., \& Davis, M. 1998, ApJ, 500, 525

Soszyński, I., Udalski, A., Szymański, M., et al. 2000, Acta Astron., 50,451

Udalski, A. 2000, Acta Astron., 50, 279

Udalski, A., Soszyński, I., Szymański, M., et al. 1999, Acta Astron., 49,1

Yi, S. K., Kim, Y.-C., \& Demarque, P. 2004, ApJS, 144, 259 